\title{
IMPORTANCIA DEL CONTEXTO SOCIAL PARA LA INVESTIGACIÓN
}

\section{IMPORTANCE OF THE SOCIAL CONTEXT FOR INVESTIGATION}

\section{Referencia del artículo}

Noriega Montufar, B. S., Rodríguez Rodríguez, R. E., López Estrada, . I. A., Buchí Guaré, C. S., Felisa Girón Hernández, M. H., \& Del Cid Flores, M. A. (2021). Importancia del Contexto Social para la Investigación. Revista Científica del Sistema de Estudios de Postgrado de la Universidad de San Carlos de Guatemala, 4(1), 77-87. DOI: https://doi.org/10.36958/sep.v4i1.77

\author{
Licda. Bertha Suceli Noriega Montufar \\ berthasuceli@hotmail.com \\ id 0000-0001-9781-6222 \\ Maestra en Docencia Universitaria \\ Licda. Rita Elena Rodríguez Rodríguez \\ bikiele@gmail.com \\ id 0000-0003-3364-8746 \\ Licenciada en Trabajo Social con Énfasis en Gerencia del \\ Desarrollo \\ Licda. Iris Aneth López Estrada \\ irislopez5713@gmail.com \\ Licenciada en Pedagogía y Administración Educativa
}

Fecha de recepción: 25/06/2021

\author{
Licda. Carolina Soledad Buchí Guaré \\ carolinabuchi@profesor.usac.edu.gt \\ id 0000-0001-9487-753X \\ Licenciada en Pedagogía y Administración Educativa \\ Licda. María Hengly Felisa Girón Hernández \\ mariahengly@profesor.usac.edu.gt \\ Licenciada en Trabajo Social en Salud Pública \\ Dr. Marco Antonio Del Cid Flores \\ delcid39@hotmail.com \\ id 0000-0002-3212-7909 \\ Doctor en educación
}

Fecha de aceptación: 08/11/2021

\section{ABSTRACT}

This essay addresses the importance of the social context for research. It starts from the premise that an individual can only be understood within the social context in which he lives.

The context is built, in the context, with the use of pertinent methodologies, methods, techniques and instruments that facilitate knowledge, with an inside look, to rescue the singularity and partialities of social processes. The subjective and experiential assessment is achieved through this interaction between the subjects who participate in the research, as objects of study and as researchers.

In the social context, knowledge is generated because it is a social product and its construction process is collective, reflecting the values, perceptions and meanings of those who build them.

It is necessary to know the social context, regardless of the approach under which the research is oriented (qualitative, quantitative or mixed) because the reality present in a context is the starting point of the scientist.

\section{KEYWORDS}

Social context, research, dimensions, phenomenon, reality

Contexto social, investigación, dimensiones, fenómeno, realidad 


\section{INTRODUCCIÓN}

La investigación es un proceso creativo y sistemático para el aumento del acervo de conocimientos, de ahí nace la importancia del contexto social en la investigación para valorar lo subjetivo como lo vivencial y conocer y comprender la realidad sociocultural a través de los mismos actores sociales. Al investigar una situación social el contexto social va a permitir conocer las necesidades, problemas y una serie de circunstancias.

Realmente esta investigación se enfoca en la necesidad de conocer el contexto social para entender y comprender la correlación de fuerzas así determinar la influencia social de los sujetos objetos de estudio para emprender desarrollo social así favorecer el cambio positivo en las relaciones de individuos, grupos e instituciones en una sociedad.

La relevancia específica de la investigación radica que el contexto social es un sistema de elementos e interrelaciones dinámicas que condicionan las distintas formas de actividad humana que a través de la interacción con la realidad se da el proceso de aprendizaje en el individuo.

Es necesario pensar en el contexto social como ese espacio que condiciona los fenómenos, problemas y relaciones de las personas que conforman el tejido social.

El ensayo se encuentra estructurado por el planteamiento, donde de forma breve se presenta la importancia del contexto social para la investigación; que es desarrollado posteriormente con ideas centrales y la reflexión, para finalmente llegar a una conclusión que es producto del análisis. Se incluyen las referencias que fueron consultadas para fundamentar teóricamente el documento.

\section{PLANTEAMIENTO}

El investigador produce conocimiento científico, a través de las lecturas que realiza al observar los escenarios sociales donde interactúan los diferentes actores de un contexto social, por lo que el contexto social es visto como un conjunto de circunstancias que enmarcan una situación o evento que afecta a uno o varios miembros de la sociedad, porque dependiendo de las circunstancias en determinado momento un mismo suceso puede afectar en varios contextos; pero su análisis, comprensión o valoración va a cambiar de acuerdo a quienes reciban la influencia directa del suceso y en quienes únicamente participen evaluando el impacto esto significa que dependiendo del contexto en que se produzca así será la comprensión e interpretación que se le dé al hecho.

Por lo que el contenido del ensayo tiene como objetivo dar a conocer la importancia que tiene para la investigación el contexto social y la capacidad que debe tener el investigador social para aprehender la realidad objetiva. El contexto social es determinante para cualquier tipo de estudio de un fenómeno o realidad. Como decía el filósofo español Ortega y Gasset, yo soy yo y mis circunstancias, es decir, solo se puede comprender a un individuo dentro del contexto social en el que vive. Como consecuencia, la historia tiene un papel preponderante en el desarrollo de los territorios.

Por eso es vital comprender que, para tener un acercamiento con la realidad, es preciso conocer el ambiente donde se desarrollan los hechos. Cómo se encuentra el tejido social, las dimensiones de lo económico, político, social, entre otros, que permitirán tener una lectura o radiografía general del contexto, en este sentido, el contexto deja de ser objeto para constituirse en sujeto del conocimiento. Se construye el contexto, con el uso 
de metodologías, métodos, técnicas e instrumentos pertinentes que faciliten obtener el conocimiento. Lo ideal es que se desarrolle un proceso participativo, para identificarlos y establecer cómo se interrelacionan en la correlación de fuerzas.

Tener la claridad de la ubicación del territorio y el conocimiento de las dinámicas del mismo es fundamental, esto permitirá identificar a los factores internos y externos, individuales y colectivos.

\section{DESARROLLO DE LA IDEA}

La investigación se basa en la comprensión de la realidad como resultado de un proceso de construcción a partir de la lógica de los diversos actores sociales, con una mirada desde adentro, para rescatar la singularidad y las parcialidades propias de los procesos sociales. Y solo es en los diferentes contextos a nivel local, regional y nacional, donde se logra hacer la valoración de lo subjetivo y lo vivencial mediante esa interacción entre los sujetos que participan en la investigación, como objetos de estudio y como investigadores donde se presta atención a lo local a lo cotidiano y cultural para comprender la lógica y el significado que tienen los procesos sociales para los propios actores sociales que son los que viven y producen la realidad sociocultural.

Es ahí donde la perspectiva holística de la investigación permite valorar los escenarios, las personas, los contextos, los grupos y las organizaciones como un todo, posibilitando estudiar a las personas en el contexto de su pasado y el de las situaciones actuales para comprender el presente. Facilitando al investigador adaptarse al contexto a investigar, y actuar de una manera pertinente en el contexto.
Se necesita conocer el contexto para comprender la correlación de fuerza que existe en el lugar, las formas de organización, niveles de organización, cultura, aspectos económicos, ideológicos, ambientales, entre otros. Lo anterior permitirá plantear desde el contexto, las preguntas pertinentes en la investigación, cuidando de no herir susceptibilidades, relacionadas al derecho consuetudinario.

De acuerdo a Raffino (2020), el contexto social puede referirse específicamente a la clase social, es decir, el lugar que un individuo ocupa dentro de los estratos de la sociedad. A la situación sociopolítica, al imaginario colectivo y, por último, a la idiosincrasia nacional, que es necesario analizarlas y comprenderlas desde cada contexto.

En el contexto, se genera el conocimiento porque es un producto social y su proceso de construcción es colectivo, allí se reflejan los valores, percepciones y significados de quienes los construyen.

Por lo tanto, es preciso conocer el contexto social, independientemente del enfoque bajo el que se oriente la investigación (cualitativo, cuantitativo o mixto) porque la realidad presente en un contexto es el punto de partida del científico que mediante la investigación le permite llegar a la ciencia, cuando observa, descubre, explica y predice aquello que lo lleva a un conocimiento sistemático de la realidad. El investigador debe ser neutral para interpretar la realidad de acuerdo con el contexto en que desarrolle los procesos para acceder al conocimiento científico.

Es determinante para cualquier tipo de investigación conocer las circunstancias que enmarcan una situación que afecta a uno, a varios individuos o una comunidad. La influencia social está presente en todos los 
ámbitos de la vida del ser humano, tanto en los juicios, comportamientos y actitudes de las personas, lo que trae como resultado que los individuos modifiquen su conducta debido a la interacción que tienen con su medio en algunos casos son evidentes cambios por la presencia del investigador social dentro de esos escenarios, que de alguna manera afecta el comportamiento de los sujetos objetos de investigación.

Toda investigación social parte de la existencia de un problema o fenómeno que requiere de su análisis, respuesta o solución. La investigación se debe situar en su contexto y entorno para conocer las múltiples realidades objetivas y subjetivas del sujeto de estudio, detectar la problemática, ponderando el relacionamiento entre ellas para permitir la viabilidad de la investigación dentro de un espacio tiempo determinado. Se puede mencionar que la función social de la investigación es el compromiso de los investigadores con los resultados y la capacidad de reflexión para el análisis crítico al abordar el problema.

En el contexto social se logran identificar datos, información y problemas de tipo educativo, ambiental, cultural, organizacional, salud, económico, vivienda, seguridad social, entre otros.

En lo educativo se identifica la cantidad de personas que cursaron educación formal e informal. Se logra conocer cuántas personas según las edades cursaron preprimaria, primaria, básico, diversificado y universidad, también el porcentaje de personas analfabetas en lectoescritura, en tecnología o en un conocimiento específico. Cuando el lugar en donde se realizará la investigación es territorialmente extenso, se puede recurrir a fuentes secundarias de investigaciones actuales, pueden ser datos de la Secretaría General de la Presidencia -SEGEPLAN-, del Instituto Nacional de Estadística -INE-, censos de las municipalidades o investigaciones de entes internacionales.

En el caso de SEGEPLAN da a conocer el Índice de Desarrollo Humano en educación y salud de cada municipio, la demografía, descripción general del municipio y la proyección. El INE a partir de los censos realizados, proporciona datos poblacionales e índices de educación. Los datos por sí solos no tendrán un significado completo, pero si se ubican en el contexto se pueden interpretar diversos fenómenos que inciden en la educación.

En lo ambiental, permite conocer la diversidad de flora y fauna del lugar, el clima, tipo de suelo, precipitaciones anuales, entre otros aspectos importantes para estar preparados ante las inclemencias del tiempo y más factores ambientales, durante el tiempo de la investigación. Hay contextos en los que hay mucha arboleda y favorece el clima, hay otros que son desérticos, los suelos son húmedos, semi húmedos, arenosos, pedregosos y mixtos.

¿Por qué la importancia de conocer este aspecto? en una ocasión un joven estudiante sin conocer el contexto social, redactó su tema de investigación relacionado a la siembra de frijol en un lugar cercano a la playa en jurisdicción de Mazatenango, Suchitepéquez, resulta que ya había pasado la fecha ideal para sembrar, pero como quería avanzar redactó que implementaría un sistema de riego, pero la variedad de frijol no fue compatible con el suelo, además que no tenía contemplado en el presupuesto todo el gasto para el sistema de riego. Es evidente que, el contexto determina cada una de las acciones que favorecen el desarrollo de las investigaciones.

En lo cultural se identifican las costumbres, tradiciones, el tejido social, formas de vida, modos de vida, maneras de comunicarse, relaciones individuales, migración, prácticas 
colectivas, idiomas, espiritualidad, creencias, patrones, hábitos, transmisión de valores de generación a generación, conocimientos propios, ideas, herramientas, procesos, entre otros. Según Máxima (2020), "la palabra cultura proviene del latín que significa cultivar, labrar o cuidar y hace referencia a cultivar el conocimiento y la educación, en el sentido de la capacidad intelectual que adquiere un pueblo o civilización" (párr. 2). Conocer la cultura, es fundamental, porque a partir de ahí se tendrá la pertinencia para relacionarse con los habitantes del lugar, al tratar a las personas con respeto.

El investigador debe adaptarse a las circunstancias culturales del contexto social, no es correcto pensar que debe ser contrario a esto. En algunas ocasiones el idioma se ha constituido en una barrera para el investigador, sin embargo, se implementan estrategias para que a través de un traductor se logre el acercamiento.

En lo organizacional se conocen aspectos que tienen que ver con el liderazgo que existe en el lugar, la participación de los habitantes, los símbolos, patrones organizacionales, distribución de tareas, costumbres, ocupación de puestos, involucramiento de la mujer, coordinación, eficiencia, eficacia, sostenibilidad de los proyectos, pertinencia organizacional, programas, proyectos, entre otros. La organización social está enfocada en atender los intereses en común, tienen un fin primordial, que es el bienestar social. La organización social se rige por normas que son definidas en cada contexto.

Como expresa Ander-Egg (1995), "la organización social denota la idea de la sociedad, en cuanto articulación de las partes como totalidad, con funciones diferentes y división social del trabajo, cuya configuración permite alcanzar los fines específicos de la vida en la sociedad". (pág. 271) En una experiencia profesional se tuvo que hacer conciencia a los hombres de la comunidad, para que las mujeres pudieran ser parte del liderazgo, porque no se les tomaba en cuenta, no fue fácil, pero se logró luego de múltiples esfuerzos.

Respecto a salud, se conocen los índices de nutrición, talla y peso de los niños, niñas y adolescentes, personas que padecen enfermedades crónicas o de transmisión sexual, personas con discapacidad, si tienen acceso a un centro de convergencia, a un centro de salud, hospital nacional, clínica privada o médico particular. Los índices de salud se encuentran reflejados en informes nacionales, por departamentos y municipios, que están relacionados a la pobreza y pobreza extrema, como un problema de raíz. Se toma en cuenta la natalidad, morbilidad y mortalidad, mujeres en edad reproductiva, entre otros.

Conocer las dimensiones de lo económico incluye el análisis de factores relacionados con el bienestar de las familias en toda área. Según las Naciones Unidas (2018), "783 millones de personas viven actualmente por debajo del umbral de pobreza internacional, con 1.90 dólares diarios" (párr. 2) y esto es vinculante a los pocos ingresos económicos familiares.

Existen múltiples metodologías para medir la pobreza, las más utilizadas a nivel mundial son la pobreza monetaria y la pobreza multidimensional. La primera capta la capacidad de un hogar de satisfacer sus necesidades básicas esenciales a través de los ingresos diarios, mientras que la segunda identifica además carencias en los ámbitos de salud, educación y nivel de vida. (Universidad Pontificia Bolivariana, 2018, párr. 3).

En los contextos es predominante conocer respecto a las viviendas y la relación de las 
familias como núcleo importante en sociedad. Aunque existen políticas sociales relacionadas a la vida digna de toda persona, muy poco se ha hecho. Cuando se conocen los contextos sociales, a través de la observación es evidente el nivel de pobreza que se puede medir a través del índice establecido en instrumentos, mediante el conocimiento del tipo de vivienda, por ejemplo, estudios socioeconómicos.

La seguridad social en los contextos sociales es preocupante, siendo importante que se conozca el avance que hay respecto a esta dimensión social, porque en algún momento como investigadores se necesitará contar con servicios relacionados.

La seguridad social se refiere a la protección que una sociedad proporciona a los individuos y los hogares para asegurar el acceso a la asistencia médica y garantizar la seguridad del ingreso, "en particular en caso de vejez, desempleo, enfermedad, invalidez, accidentes del trabajo, maternidad o pérdida del sostén de familia". (Organización Internacional del Trabajo -OIT-, 2001, pág. 1) La importancia del contexto en la investigación reside en que toda investigación inicia con el estudio del contexto para conocer los elementos y factores que favorecen u obstaculizan los diversos procesos que en él se producen.

El contexto de la investigación se delimita a través del interés del investigador que mediante un proceso metodológico se dirige a la búsqueda del conocimiento a través de la objetividad y creatividad, de donde emergen las ideas, se van entretejiendo y articulando hasta construir conocimientos como producto de la aplicación de un método para interpretar esa realidad del objeto o fenómeno de estudio.

\section{CONCLUSIÓN}

El contexto social es fundamental porque las ideas y prácticas que surgen en un determinado contexto social, político, económico y cultural pueden no aplicar a un contexto con condiciones diferentes.

El contexto social se constituye en el laboratorio de los investigadores sociales, en él están incluidos los fenómenos que estudia y al cual emergen investigadores de diferentes disciplinas sociales para que a través de sus diferentes procesos de investigación le permita interpretar la realidad y pueda describir, explicar y predecir la realidad del futuro del fenómeno que investiga, para asegurar el desarrollo histórico de un objeto de estudio enfocado de acuerdo a la necesidad del desarrollo social.

\section{REFERENCIAS}

Ander-Egg, E. (1995). Diccionario de trabajo social. Buenos Aires: AR: LUMEN.

Guillen, M. (2014) Contexto Social. Memoria Académica. Costa Rica. Máxima, J. (21 de mayo de 2020). Significado de cultura. Recuperado el 15 de mayo de 2021, de https://www.caracteristicas. co/cultura/.

Organización de las Naciones Unidas. (10 de diciembre de 2018). Dimensiones de lo económico. Recuperado el 21 de febrero de 2021, de https: / /www.ohchr.org/ SP/Professionallnterest/Pages/CAT. aspx

Organización Internacional del Trabajo. (2001). Conferencia Internacional sobre Seguridad Social. Recuperado el 15 de mayo de 2021, de https://www.ilo.org/wcmsp5/groups/ public/---dgreports/---dcomm/documents/publication/wcms_067592.pdf 
Pérez, M. (2010) El Contexto Social. Tesis Doctoral en Xarxa. Barcelona.

Raffino, M. (08 de julio de 2020). Contexto social. Recuperado el 08 de mayo de 2021, de https://contextosocial-3/\#: :text $=$ La\%20psicolog\%C $3 \% A D a \% 200 \% 20$ sicolog\%C3\%ADa\%20es, durante\%20momentos\%20y\%20situaciones\%20determinadas.

Universidad Pontificia Bolivariana. (2018). Dimensión económica. Recuperado el 15 de mayo de 2021, de https: / /www.upb. edu.co/es/sostenibilidad/que-es-dimension-economica-o

\section{SOBRE AUTORES}

\section{Licda. Bertha Suceli Noriega Montufar}

Es Licenciada en Trabajo Social, Maestra en Docencia Universitaria, Maestrando de Gestión Comunitaria para el Desarrollo Humano Local, asesora consultora educativa y social, capacitadora de temas educativos y sociales, ha asesorado tesis de grado y postgrado en la USAC y universidades privadas.

\section{Licda. Rita Elena Rodríguez Rodríguez}

Es Profesora en la Carrera Trabajo Social en el Centro Universitario de Sur Occidente desde hace 13 años. Licenciada en Trabajo Social con Énfasis en Gerencia del Desarrollo, pensum cerrado en la maestría Antropología Social, Maestrando de Gestión Comunitaria para el Desarrollo Humano Local.

\section{Licda. Iris Aneth López Estrada}

Es maestra de Educación Primaria Urbana, Profesora de Enseñanza Media, Licenciada en Pedagogía y Administración Educativa, Cierre de Pensum en Trabajo Social, Maestrando de Gestión Comunitaria y Desarrollo Local. Integrante de la Directiva de la Asociación Esperanza para el Desarrollo Comunitario ASESDECO.

\section{Licda. Carolina Soledad Buchí Guaré}

Es Profesora de licenciatura en Pedagogía CUNSUROC, tiene cierre de Pensum de la Maestría de Antropología Social, Maestrando de Gestión Comunitaria Para el Desarrollo Humano Local. Reconocimiento por el Instituto de Investigación y Desarrollo de Sur Occidente (IIDESO) a la mejor tesis 2004-2005, ha revisado trabajos de tesis de grado.

\section{Licda. Maria Hengly Felisa Girón Her- nández}

Es Profesora en la Carrera Trabajo Social en el Centro Universitario de Sur Occidente desde el 2007. Licenciada en Trabajo Social en Salud Pública, con especialidad en VIH, Maestrando de Gestión Comunitaria para el Desarrollo Humano Local.

\section{Dr. Marco Antonio Del Cid Flores}

Dr. Marco Antonio Del Cid Flores, Licenciado en Zootecnia, Maestro en docencia Universitaria, Doctor en educación, Doctor en Investigación Social, Postdoctor en Ciencias de la educación con especialidad en liderazgo y Gestión de la Educación Universitaria; Postdoctor En Ciencias de la Educación con Especialidad en Psicología Educativa. Director del Departamento de Estudios de Postgrados del Centro Universitario de Suroccidente -Cunsuroc-. Investigador Postdoctoral en Universitario Tecnológico Terra At Mundi Universitam De Tijuana. Asesor de mas de 300 tesis a nivel de licenciatura, maestría y doctorado. 


\section{Conflicto de intereses \\ Declara no tener ningún conflicto de intereses.}

Declaración de consentimiento informado

El estudio se realizó, respetando el Código de ética y buenas prácticas editoriales de publicación.

\section{Derechos de uso}

Copyright $\odot 2021$ por Bertha Suceli Noriega Montufar, Rita Elena Rodiguez Rodríguez, Iris Aneth López Estrada, Carolina Soledad Buchí Guaré, María Hengly Felisa Girón Hernández y Marco Antonio Del Cid Flores

Este texto está protegido por una licencia Creative Commons 4.0. Internacional

Usted es libre para compartir, copiar y redistribuir el material en cualquier medio o formato y adaptar el documento, remezclar, transformar y crear a partir del material para cualquier propósito, incluso comercialmente, siempre que cumpla la condición de atribución: usted debe reconocer el crédito de una obra de manera adecuada, proporcionar un enlace a la licencia, e indicar si se han realizado cambios. Puede hacerlo en cualquier forma razonable, pero no de forma tal que sugiera que tiene el apoyo del licenciante o lo recibe por el uso que hace. 